\title{
MID-INFRARED DIAGNOSTICS OF LINERS
}

\author{
E. Sturm, ${ }^{1}$ D. Rupke, ${ }^{2}$ A. Contursi, ${ }^{1}$ D.-C. Kim, ${ }^{2}$ D. Lutz, ${ }^{1}$ H. Netzer, ${ }^{1,3}$ S. Veilleux, ${ }^{2}$ R. Genzel, ${ }^{1}$ M. Lehnert, ${ }^{1}$ \\ L. J. Tacconi, ${ }^{1}$ D. Maoz, ${ }^{3}$ J. Mazzarella, ${ }^{4}$ S. Lord, ${ }^{4}$ D. Sanders, ${ }^{5}$ and A. Sternberg ${ }^{3}$ \\ Received 2006 July 11; accepted 2006 October 24; published 2006 November 27
}

\begin{abstract}
We report results from the first mid-infrared spectroscopic study of a comprehensive sample of 33 LINERs, observed with the Spitzer Space Telescope. We compare the properties of two different LINER populations: infrared-faint LINERs, with LINER emission arising mostly in compact nuclear regions, and infrared-luminous LINERs, which often show spatially extended (non-AGN) LINER emission. We show that these two populations can be easily distinguished by their mid-infrared spectra in three different ways: (1) their mid-IR spectral energy distributions (SEDs), (2) the emission features of polycyclic aromatic hydrocarbons (PAHs), and (3) various combinations of IR fine-structure line ratios. IR-luminous LINERs show mid-IR SEDs typical of starburst galaxies, while the mid-IR SEDs of IR-faint LINERs are much bluer. PAH flux ratios are significantly different in the two groups. Fine-structure emission lines from highly excited gas, such as [O IV], are detected in both populations, suggesting the presence of an additional AGN in a large fraction of IR-bright LINERs as well, which contributes little to the combined mid-IR light. The two LINER groups occupy different regions of mid-infrared emissionline excitation diagrams. The positions of the various LINER types in our diagnostic diagrams provide important clues regarding the power source of each LINER type. Most of these mid-infrared diagnostics can be applied at low spectral resolution, making AGN- and starburst-excited LINERs distinguishable at high redshifts as well.
\end{abstract}

Subject headings: galaxies: active — infrared: galaxies

Online material: color figures

\section{INTRODUCTION}

Since their identification as a class of galactic nuclei more than 25 years ago (Heckman 1980), the nature of low-ionization nuclear emission-line regions (LINERs) has remained controversial. Their optical spectra are characterized by enhanced narrow emission lines of low-ionization species, quite distinct from those of both $\mathrm{H}$ II regions and classical active galactic nuclei (AGNs). They are found in one-third to one-half of all types nearby galaxies (e.g., Ho et al. 1997). In many LINERs the emission is concentrated near the nucleus (a few times $100 \mathrm{pc}$; e.g., Pogge et al. 2000), but in others it extends over larger regions, up to a few kiloparsecs (Veilleux et al. 1995). There is substantial evidence that many LINERs are powered by accretion onto massive black holes and that these objects, due to low accretion rates, constitute the low-luminosity end of the AGN class (Quataert 2001; Kewley et al. 2006). If many LINERs at low and high redshifts are indeed low-luminosity AGNs, this would have a significant impact on major issues in astronomy such as the growth history of central black holes and the relation of AGNs to galaxy formation and evolution.

Alternative scenarios for LINER excitation mechanisms have been suggested. Models focusing on photoionization by the central AGN continuum (e.g., Ferland \& Netzer 1983; Groves et al. 2004) have been complemented by stellar photoionization modeling (e.g., Barth \& Shields 2000) and observations (e.g., Maoz et al. 1998) showing that, under certain conditions, a young starburst can also excite a LINER. In many cases LINER-like emission is also observed on larger spatial scales; these "ex-

\footnotetext{
${ }^{1}$ Max-Planck-Institut für extraterrestrische Physik (MPE), Postfach 1312, D-85741 Garching, Germany; sturm@mpe.mpg.de.

${ }^{2}$ Department of Astronomy, University of Maryland, College Park, MD 20742.

${ }^{3}$ School of Physics and Astronomy, Tel Aviv University, Ramat Aviv, Tel Aviv 69978, Israel.

${ }^{4}$ IPAC/California Institute of Technology, MS 100-22, Pasadena, CA 91125.

${ }^{5}$ Institute for Astronomy, University of Hawaii, 2680 Woodlawn Drive, Honolulu, HI 96822.
}

tended" LINERs tend to be associated with high infrared luminosity (see § 2). In addition to nuclear (black hole or stellar) photoionization, these sources can be ionized by shock heating through cloud collisions induced by accretion, galaxy interactions, mergers, or starburst-driven winds (e.g., Shull \& McKee 1979; Veilleux \& Osterbrock 1987; Dopita \& Sutherland 1995).

Determining which LINERs are photoionized by a hard, nuclear power source and which are excited by other ionization processes is crucial for understanding not only the local LINER population but also AGNs and starbursts at high redshifts, if the fraction of LINERs among high- $z$ galaxies is as high as in the local universe. Galaxies with high infrared luminosities and galaxy interactions are much more common at high redshifts than at $z \approx 0$ (e.g., Pérez-González et al. 2005). Whether or not these galaxies host dominant AGNs is an open question. X-ray and radio observations help to identify AGNs in the nuclei of these galaxies. However, they may face problems in deriving the relative contributions of AGNs and starbursts to the luminosity of those objects, in particular, for close to Compton-thick AGNs (e.g., NGC 6240; Lutz et al. 2003). In order to help resolve these problems, we have performed a mid-infrared spectroscopic study that is based on a large sample of infrared-faint and infraredluminous LINERs. This study is the first of its kind, since only a few LINERs were observed with the Infrared Space Observatory $(I S O)$ as part of various projects with differing scientific goals (Satyapal et al. 2004), resulting in a sparse collection of mid-IR LINER line detections of rather low signal-to-noise ratio $(\mathrm{S} / \mathrm{N})$ in a heterogeneous sample. Comprehensive mid-infrared spectroscopic studies of LINERs have become possible only now with Spitzer.

\section{SAMPLE SELECTION, OBSERVATIONS, AND DATA PROCESSING}

We have based our selection of LINERs on the infrared luminosity of low-redshift objects. Most of the IR-faint LINERs $\left(L_{\mathrm{IR}} / L_{B} \lesssim 1\right.$, with $L_{\mathrm{IR}}$, the $8-1000 \mu \mathrm{m}$ luminosity, defined as in 
Sanders \& Mirabel 1996) studied in the literature have compact nuclei (e.g., Pogge et al. 2000), consistent with photoionization from a central source. This central source is very likely an AGN, since most of these IR-faint LINERs have compact nuclear Xray sources but no extended X-ray sources (e.g., Carrillo et al. 1999; Satyapal et al. 2004). On the other hand, IR-luminous LINERs $\left(L_{\mathrm{IR}} / L_{B} \gtrsim 1\right)$ often also show compact hard X-ray cores indicative of an AGN, but in addition they often contain multiple off-nuclear X-ray point sources. There is evidence of extended LINER emission in a number of LINERs in the Revised Bright Galaxy Survey (RBGS; see Veilleux et al. 1995). A famous example is the extended LINER emission in NGC 6240 (Veilleux et al. 2003). Monreal-Ibero et al. (2006) also show integral-field unit $[\mathrm{N}$ II] $/ \mathrm{H} \alpha$ excitation maps of IR-luminous LINERs showing extended LINER emission. Our two subsamples of different IRluminosity therefore represent statistically different LINER populations (nuclear vs. extended LINER emission).

The sample is summarized in Table 1 . We selected 16 IRluminous LINERs from the RBGS and 17 IR-faint LINERs from Ho et al. (1997). The IR-faint LINERs are further subdivided into five type 1 and six type 2 LINERs, and in addition six "transition" objects (see Ho et al. 1997 and our $§$ 3.1). The infrared-bright LINERs are all type 2 . With a median $L_{\mathrm{IR}}$ of 11.31 $L_{\odot}$, the IR-luminous LINERs are much more luminous than the IR-faint LINERs in the IR, and there is no significant difference between the IR-faint subclasses (L1: $8.92 L_{\odot}$; L2: $9.27 L_{\odot} ; \mathrm{T} 2: 9.31 L_{\odot}$ ). For further multiwavelength information for all our objects, we refer to Carrillo et al. (1999). Table 1 also lists $L_{\mathrm{IR}} / L_{B}$ ratios. The median ratios are 106 and 1.8 for the IR-bright and IR-faint LINERs, respectively.

The data were obtained with the Infrared Spectrograph (IRS; Houck et al. 2004) on board Spitzer in staring mode. Highresolution spectra covering 10-37 $\mu \mathrm{m}$ (modules $\mathrm{SH}$ and $\mathrm{LH}$ ) are complemented by low-resolution spectra in the 5-15 $\mu \mathrm{m}$ range (modules SL2 and SL1). Our data reduction process started with the two-dimensional Basic Calibrated Data products from the Spitzer pipeline (ver. S12). We used our own IDL routines to perform deglitching, and we used SMART (Higdon et al. 2004) for the final spectrum extraction.

All our targets are nearby $(D \leq 120 \mathrm{Mpc})$, but on average the IR-faint LINERs have lower redshifts (median $z=0.004$ ) than the IR-bright objects (median $z=0.023$ ). We carefully checked that aperture effects (extended objects at different distances, and fluxes from different aperture sizes in the different IRS modules) do not significantly affect the spectra and our conclusions. For most of the targets, the 12 and $25 \mu \mathrm{m}$ flux densities agree quite well with the IRAS values (the worst deviation is a factor 2 in NGC 5371, a nearby, IR-faint LINER that is dominated by mid-IR emission from the spiral arms); flux density discrepancies in the overlapping wavelength region between subspectra obtained with different aperture sizes are of the same order; and the profiles of most of the two-dimensional spectral images do not deviate significantly from stellar (point-source) profiles (i.e., the observed flux in most of the objects is coming from a nuclear region of $0.3 \mathrm{kpc}$ [median] diameter). We did not apply an extended source correction to the flux calibration, and we used full width apertures for the spectral extraction. More details about the sample, the observations, and the data processing will be described in a forthcoming paper (D. Rupke et al. 2006, in preparation).

\section{MID-INFRARED PROPERTIES OF LINERS}

Our new Spitzer observations provide us with three ways to distinguish among the various subclasses of LINERs: the mid-
TABLE 1

THE LINER SAMPLE

\begin{tabular}{|c|c|c|c|}
\hline Object Name & Type & $L_{\mathrm{IR}}^{\mathrm{a}}$ & $L_{\mathrm{IR}} / L_{B}^{\mathrm{b}}$ \\
\hline \multicolumn{4}{|c|}{ Revised Bright Galaxy Survey (Veilleux et al. 1995) } \\
\hline UGC 556 & L2 & 10.91 & 106 \\
\hline UGC 2238 & L2 & 11.35 & 93 \\
\hline IRAS $02438+2122$ & L2 & 11.20 & 205 \\
\hline NGC 1204 & L2 & 10.96 & 113 \\
\hline IRAS $05187-1017$ & L2 & 11.31 & 229 \\
\hline NGC $4666 \ldots \ldots \ldots \ldots$ & L2 & 10.44 & 29 \\
\hline NGC $4922 \ldots .$. & L2 & 11.31 & 103 \\
\hline UGC $8387 \ldots$. & L2 & 11.65 & 135 \\
\hline NGC 5104 & L2 & 11.19 & 79 \\
\hline NGC $5218 \ldots \ldots \ldots \ldots$ & L2 & 10.64 & 21 \\
\hline I Zw $107 \ldots \ldots$ & L2 & 11.89 & $\ldots$ \\
\hline IRAS $15335-0513 \ldots \ldots$ & L2 & 11.42 & $\ldots$ \\
\hline IRAS $16164-0746 \ldots \ldots$ & L2 & 11.44 & 210 \\
\hline ESO 602-G025 & L2 & 11.33 & 47 \\
\hline Zw $453.062 \ldots \ldots \ldots \ldots$ & L2 & 11.38 & 116 \\
\hline NGC $7591 \ldots \ldots \ldots \ldots$ & L2 & 11.12 & 47 \\
\hline \multicolumn{4}{|c|}{ Ho et al. (1997) } \\
\hline NGC 404 . & L2 & 7.83 & 1.1 \\
\hline NGC 3245 & $\mathrm{~T} 2$ & 9.48 & 1.8 \\
\hline NGC 3507 & $\mathrm{~L} 2$ & & .. \\
\hline NGC $3642 \ldots$ & L1 & 9.65 & 1.4 \\
\hline NGC $3884 \ldots \ldots \ldots \ldots$ & L1 & $\ldots$ & $\ldots$ \\
\hline NGC $3898 \ldots \ldots \ldots \ldots$ & $\mathrm{T} 2$ & 9.13 & 0.6 \\
\hline NGC $3998 \ldots \ldots \ldots \ldots$ & L1 & 8.66 & 0.6 \\
\hline NGC $4036 \ldots \ldots \ldots \ldots$ & L1 & 9.18 & 0.5 \\
\hline NGC $4192 \ldots \ldots \ldots \ldots$ & $\mathrm{T} 2$ & 9.72 & 4.7 \\
\hline NGC 4278 & L1 & 8.50 & 0.5 \\
\hline NGC $4419 \ldots \ldots \ldots \ldots$ & $\mathrm{T} 2$ & 9.91 & 11 \\
\hline NGC $4435 \ldots \ldots \ldots \ldots$ & $\mathrm{T} 2 / \mathrm{H}$ & 9.09 & 1.9 \\
\hline NGC 4457 & L2 & 9.27 & 4.3 \\
\hline NGC $4486 \ldots \ldots \ldots \ldots$ & L2 & 8.95 & 0.1 \\
\hline NGC $5371 \ldots \ldots \ldots \ldots$ & L2 & $10.59^{\mathrm{c}}$ & 4.9 \\
\hline NGC $6500 \ldots \ldots \ldots \ldots$ & L2 & 9.74 & 3.0 \\
\hline NGC $7177 \ldots \ldots \ldots \ldots$ & $\mathrm{T} 2$ & $\ldots$ & $\ldots$ \\
\hline
\end{tabular}

${ }^{\text {a }}$ The logarithm of the $8-1000 \mu \mathrm{m}$ luminosity is in units of solar luminosity.

${ }^{\mathrm{b}} L_{B}$ is from the $B$ magnitudes in Carrillo et al. (1999).

c The nuclear LINER is from Ho et al. (1997), with much of the mid-IR luminosity coming from spiral arms outside the IRS apertures; hence, it is treated as an IR-faint LINER.

infrared SEDs, the PAH emission features, and various combinations of IR fine-structure line ratios. These are illustrated and discussed below. Figure 1 shows the average spectra of both subsamples (with the IR-faint LINERs further divided into the three subgroups type 1, type 2, and transition objects; see $\S 2$ ). As described in $\S 2$, the IR-faint LINERs, including the transition objects, are much less luminous in the IR than the IR-luminous ones. Before averaging, the individual spectra have been normalized at $19 \mu \mathrm{m}$. The average spectra show clear differences among the subgroups. Because of a low dispersion within the two major groups (IR-luminous and IRfaint), almost all of our individual spectra can be unambiguously assigned to one of these two groups (Fig. 2).

\subsection{SED Shape}

The mid-infrared SEDs of IR-faint LINERs are clearly flatter (i.e., bluer) than their IR-luminous counterparts. No strong absorption features due to silicates (at 9.7 and $18 \mu \mathrm{m}$ ) or ices (at various wavelengths) are seen in either subsample. Only one IR-faint LINER, NGC 3998, reveals strong silicate emission (Sturm et al. 2005). In contrast, the mid-infrared SEDs of infrared-bright LINERs generally look very similar to those of starburst galaxies (e.g., Sturm et al. 2000). There are interesting trends shown in Figure 1. The type 1 and type 2 spectra are very similar, but below $\sim 15 \mu \mathrm{m}$ the average type 1 spectrum 


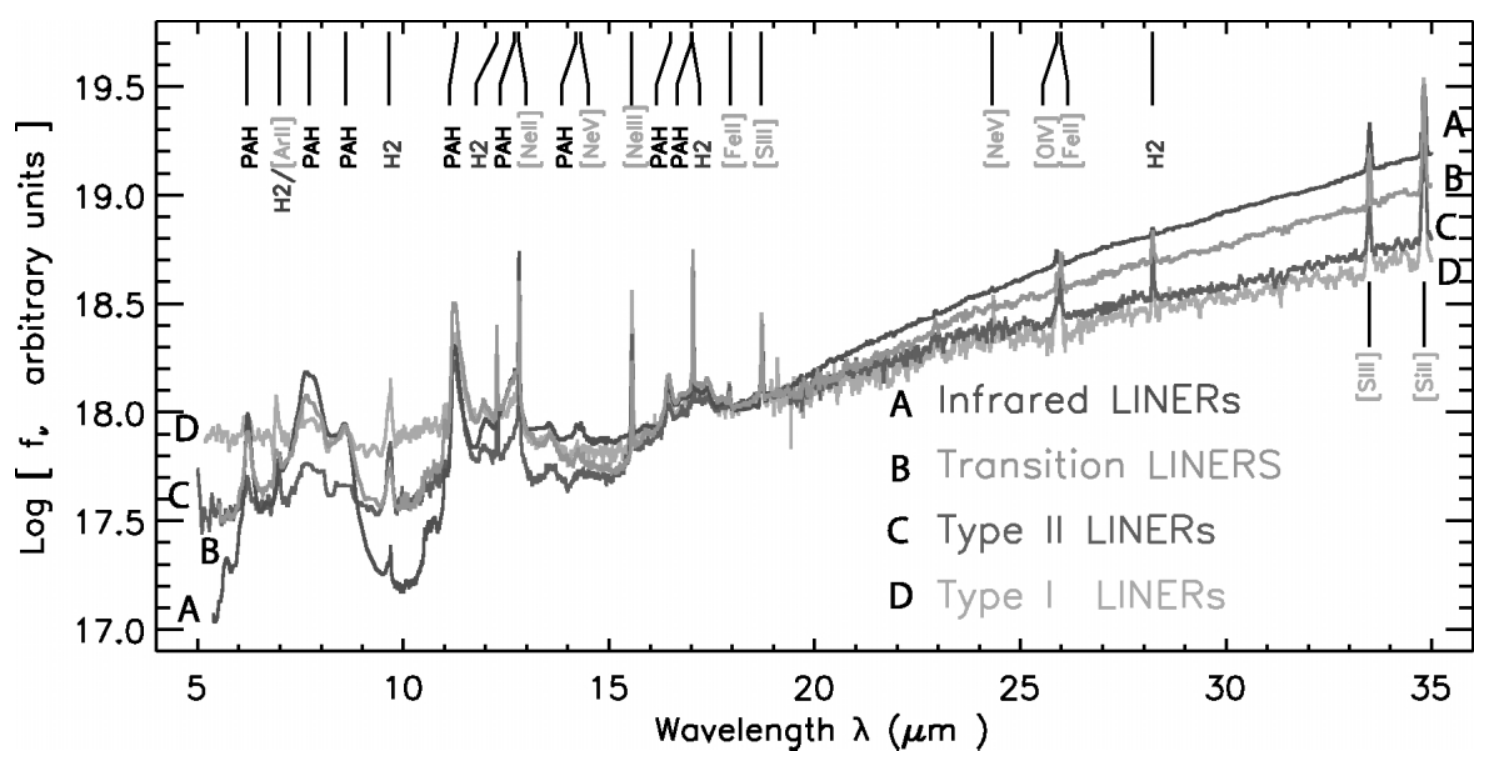

FIG. 1.-Composite spectra (normalized at $19 \mu \mathrm{m}$ ) of the 16 IR-luminous LINERs (black line) and the IR-faint LINERs (light gray line: five type 1 LINERs, dark gray line: six type 2 LINERs, gray line: six transition types). [See the electronic edition of the Journal for a color version of this figure.]

has an additional warm (dust) component. The average transition LINER lies in between the type 1/type 2 objects and the IR-bright LINERs. These transition objects are classified as objects with [O $\mathrm{O}$ I] strengths intermediate between those of $\mathrm{H}$ II nuclei and LINERs, which could be explained either by a dilution of "true" LINER spectra with circumnuclear star formation or by stellar photoionization (see Ho et al. 1997).

The differences in the mid-IR SEDs of the two major LINER types are quantified in Figure $2 a$ : in a diagram of the continuum flux ratios $f_{\nu}(15 \mu \mathrm{m}) / f_{\nu}(6 \mu \mathrm{m})$ versus $f_{\nu}(30 \mu \mathrm{m}) / f_{\nu}(6 \mu \mathrm{m})$, IR-faint and IR-bright galaxies are nicely separated. The warmer spectrum of (some of) the type 1 LINERs compared to the type 2 LINERs (see also Fig. 1) could be the signature of a warm AGN continuum (as expected in a comparison of type 1 and type 2 AGNs, assuming the AGN unification model holds for LINERs). Maoz et al. (2005) have found a similar trend in the UV continuum of IR-faint LINERs. In addition, in some of the weaker, nearby IR-faint LINERS, photospheric emission from old stellar populations in the centers of the host galaxies may contribute significantly to the measured continuum in the range from 5 to $\sim 8 \mu \mathrm{m}$. Using the same scaling factor as Lutz et al. (2004) to extrapolate from the $K$ band to the mid-IR, and assuming that most of the $K$-band flux in our IRS aperture is stellar, we conclude that this might indeed be the case for some of the IR-faint
LINERs. Stellar contribution is probably not the sole explanation, however, because it could hardly explain the difference between (the average) type 1 and type 2 LINERs (the $K$-band/15 $\mu \mathrm{m}$ ratios are similar in both types), and because the typical signature of such a component, a decreasing continuum in the 5-8 $\mu \mathrm{m}$ range, is not prominent in most of the individual spectra.

\subsection{PAH Ratios}

Figure 1 also shows significant differences in the PAH features in the spectra of the two LINER groups. In IR-bright LINERs, the PAH spectrum is very similar to starburst galaxies. IR-faint LINERs have very weak PAH features in the $5-10 \mu \mathrm{m}$ range but strikingly strong $11.2 \mu \mathrm{m}$ features. We note that, in principle, a higher degree of dilution by an additional warm dust component in the IR-faint LINERs could cause errors in the flux measurements of PAHs in the 5-10 $\mu \mathrm{m}$ range, if the continuum has a structure of similar width as the PAHs. For instance, strong silicate emission or absorption around $10 \mu \mathrm{m}$, if not accounted for, could lead to systematically false flux values of broad PAH features like the 7.7/8.6 PAH complex. Such an effect, however, should not play a significant role in our measurements of the relatively narrow, well-defined PAHs at 6.2, 11.2, and $12.7 \mu \mathrm{m}$. Based on the flux ratios of these PAH features at 6.2, 11.2, and
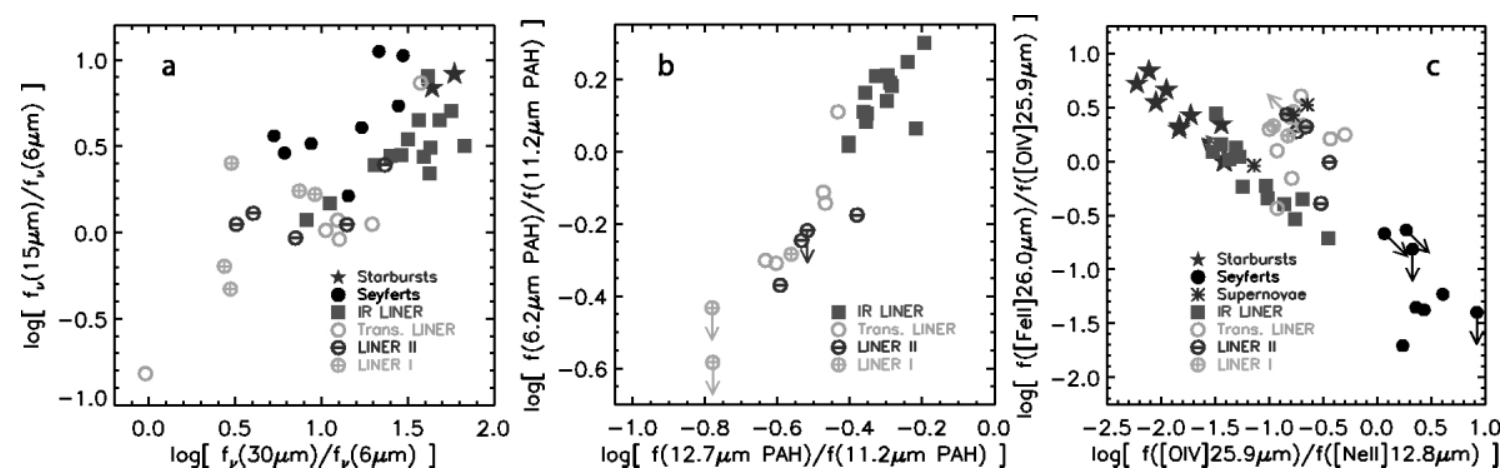

FIG. 2.-Mid-infrared diagnostic diagrams to distinguish between IR-luminous and IR-faint LINERs. In panel $a$, the Seyfert data are from Weedman et al. (2005), and the starburst data are from Sturm et al. (2000; M82) and Brandl et al. (2004; NGC 7714). The Seyfert and starburst data in panel $c$ are from Sturm et al. (2002) and Verma et al. (2003). Error bars in all three panels are comparable to the symbol sizes. [See the electronic edition of the Journal for a color version of this figure.] 
$12.7 \mu \mathrm{m}$, we present in Figure $2 b$ another diagnostic diagram for the distinction between IR-bright and IR-faint LINERs. Hony et al. (2001, their Fig. 5) have constructed such a diagram using ISO spectra of galactic $\mathrm{H}$ II regions, young stellar objects, and evolved stars/reflection nebulae. They found that the strongest 11.2 $\mu \mathrm{m}$ PAH features (lowest 12.7/11.2 and 6.2/11.2 ratios) are produced in evolved stars, while $\mathrm{H}$ II regions have the highest 12.7/11.2 (and 6.2/11.2) ratios. They attributed this to a different degree of ionization (the $11.2 \mu \mathrm{m}$ PAH carriers are neutral, those of the 6.2 and 12.7 features are ionized; see also Joblin et al. $1996)$ as well as a processing of PAHs from large $(11.2 \mu \mathrm{m})$ to smaller $(12.7 \mu \mathrm{m})$ carriers. This could imply a less ionizing environment in the IR-faint LINERs than in the IR-bright ones, or significant differences in PAH formation and destruction.

\subsection{Emission-Line Ratios}

The mid-infrared LINER spectra are rich in fine-structure emission lines. We have detected strong [O IV] lines, i.e., highly ionized gas, in both LINER subsamples, suggestive of the presence of an AGN in $\sim 90 \%$ of the LINERs. The presence of a weak AGN in many of the IR-luminous LINERs is consistent with the compact hard X-ray cores that many IR-bright LINERs show (see $\S 2$ ) and is further supported by the detection of $[\mathrm{Ne} v]$ in about half of these sources. This detection rate is certainly a lower limit due to the limited $\mathrm{S} / \mathrm{N}$ in the $[\mathrm{Ne} \mathrm{v}]$ spectra. In order to explore differences in relative line strengths and physical properties, we have constructed mid-infrared diagnostic diagrams involving various combinations of these finestructure emission lines. Figure $2 c$ is an example of such a diagram using the line ratios of [Fe II] $26.0 \mu \mathrm{m} /[\mathrm{O}$ IV] $25.9 \mu \mathrm{m}$ and [O IV] $25.9 \mu \mathrm{m} /[\mathrm{Ne}$ II] $12.8 \mu \mathrm{m}$. Lutz et al. (2003) used this diagram to distinguish between excitations by early-type stars, AGNs, and shocks. Starburst galaxies, Seyfert galaxies, and supernova remnants (SNRs) are clearly separated in this line ratio plane. Interestingly, IR-luminous and IR-faint LINERs are easily distinguishable in this diagram: the IR-luminous LINERs all lie on a linear relation connecting starburst galaxies and Seyfert galaxies, which might be explained by a minor AGN contribution in addition to the star-forming regions (see mixing lines in Lutz et al. 2003). The positions of the IR-luminous LINERs in this diagram are an indication that the weak AGNs in these objects are more Seyfert-like, i.e., not responsible for the IR-faint LINER emission. Surprisingly, the IR-faint LINERs are clearly off this line and lie in a region populated by SNRs. The reason for the differences between the two classes (and between IR-faint LINERs and Seyfert galaxies) is so far uncertain. The role of the hardness of the radiation spectrum and of the ionization parameter, as well as the influence of shocks in both LINER classes, has to be further examined. Can, e.g., photoionized spectra for certain hardness and ionization parameters reach the "SNR" region in Figure $2 c$ ?

\section{CONCLUSIONS AND OUTLOOK}

Our systematic study of LINERs with Spitzer confirms that IR-luminous sources of this group are very different in their properties from IR-faint LINERs. IR-luminous LINERs have infrared SEDs that are similar to those of starburst galaxies, and they are situated in different regions of several diagnostic diagrams than IR-faint LINERs. While this has been suspected from previous multiwavelength studies, the new mid-IR data provide us with the cleanest way to separate those groups. Many of these differences are also visible at low spectral resolution, and thus at high redshift, making our discovery an important cosmological tool for discerning the role of AGNs in galaxy formation and evolution. A more detailed investigation of this issue and a quantification of the relative contributions from AGNs and star formation to the LINER spectra require a careful disentangling of the spectra into the various components (stellar, $\mathrm{H}$ II region, AGN, etc.), involving template fitting methods. Furthermore, for an understanding of the emission-line properties, detailed photoionization modeling is required. This will be the subject of a forthcoming paper (D. Rupke et al. 2006, in preparation).

This work is based on observations made with the Spitzer Space Telescope, which is operated by the Jet Propulsion Laboratory, California Institute of Technology, under a contract with NASA. Support for this work was provided by NASA through an award issued by JPL/Caltech. A. S. and H. N. thank the Israel Science Foundation for support grants 221/03 and 232/03. H. N. acknowledges support by the Humboldt Foundation and thanks the host institution MPE.

\section{REFERENCES}

Barth, A. J., \& Shields, J. C. 2000, PASP, 112, 753

Brandl, B., et al. 2004, ApJS, 154, 188

Carrillo, R., Masegosa, J., Dultzin-Hacyan, D., \& Ordoñez, R. 1999, Rev. Mex. AA, 35, 187

Dopita, M. A., \& Sutherland, R. S. 1995, ApJ, 455, 468

Ferland, G. J., \& Netzer, H. 1983, ApJ, 264, 105

Groves, B. A., Dopita, M. A., \& Sutherland, R. S. 2004, ApJS, 153, 9

Heckman, T. M. 1980, A\&A, 87, 152

Higdon, S. J. U., et al. 2004, PASP, 116, 975

Ho, L. C., Filippenko, A. V., \& Sargent, W. L. W. 1997, ApJS, 112, 315

Hony, S., Van Kerckhoven, C., Peeters, E., Tielens, A. G. G. M., Hudgins, D. M., \& Allamandola, L. J. 2001, A\&A, 370, 1030

Houck, J. R., et al. 2004, ApJS, 154, 18

Joblin, C., Tielens, A. G. G. M., Geballe, T. R., \& Wooden, D. H. 1996, ApJ, 460, L119

Kewley, L. J., Groves, B., Kauffmann, G., \& Heckman, T. 2006, MNRAS, 372, 961

Lutz, D., Maiolino, R., Spoon, H. W. W., \& Moorwood, A. F. M. 2004, A\&A, 418, 465

Lutz, D., Sturm, E., Genzel, R., Spoon, H. W. W., Moorwood, A. F. M., Netzer, H., \& Sternberg, A. 2003, A\&A, 409, 867

Maoz, D., Koratkar, A., Shields, J. C., Ho, L. C., Filippenko, A. V., \& Sternberg, A. $1998, \mathrm{AJ}, 116,55$
Maoz, D., Nagar, N. M., Falcke, H., \& Wilson, A. S. 2005, ApJ, 625, 699

Monreal-Ibero, A., Arribas, S., \& Colina, L. 2006, ApJ, 637, 138

Pérez-González, P. G., et al. 2005, ApJ, 630, 82

Pogge, R. W., Maoz, D., Ho, L. C., \& Eracleous, M. 2000, ApJ, 532, 323

Quataert, E. 2001, in ASP Conf. Ser. 224, Probing the Physics of Active Galactic Nuclei, ed. B. M. Peterson, R. W. Pogge, \& R. S. Polidan (San Francisco: ASP), 71

Sanders, D. B., \& Mirabel, I. F. 1996, ARA\&A, 34, 749

Satyapal, S., Sambruna, R. M., \& Dudik, R. P. 2004, A\&A, 414, 825

Shull, J. M., \& McKee, C. F. 1979, ApJ, 227, 131

Sturm, E., Lutz, D., Tran, D., Feuchtgruber, H., Genzel, R., Kunze, D., Moorwood, A. F. M., \& Thornley, M. D. 2000, A\&A, 358, 481

Sturm, E., Lutz, D., Verma, A., Netzer, H., Sternberg, A., Moorwood, A. F. M., Oliva, E., \& Genzel, R. 2002, A\&A, 393, 821

Sturm, E., et al. 2005, ApJ, 629, L21

Veilleux, S., Kim, D.-C., Sanders, D. B., Mazzarella, J. M., \& Soifer, B. T. 1995, ApJS, 98, 171

Veilleux, S., \& Osterbrock, 1987, ApJS, 63, 295

Veilleux, S., Shopbell, P. L., Rupke, D. S., Bland-Hawthorn, J., \& Cecil, G. 2003, AJ, 126, 2185

Verma, A., Lutz, D., Sturm, E., Sternberg, A., Genzel, R., \& Vacca, W. 2003, A\&A, 403, 829

Weedman, D. W., et al. 2005, ApJ, 633, 706 